\title{
Genetic therapies for cystic fibrosis lung disease
}

\section{Stephen L. Hart ${ }^{1 *}$ and Patrick T. Harrison ${ }^{2}$}

1. Experimental and Personalised Medicines Section, Genetics and Genomic Medicine Programme, UCL Great Ormond Street Institute of Child Health, 30 Guilford St, London, WC1N $1 E H, U K$

2. Department of Physiology, BioSciences Institute, University College Cork, Cork, Ireland

*Corresponding author; s.hart@ucl.ac.uk

\begin{abstract}
Gene therapy for cystic fibrosis (CF) has been the subject of intense research over the last twenty-five years or more, using both viral and liposomal delivery methods, but so far without the emergence of a clinical therapy. New approaches to CF gene therapy involving recent improvements to vector systems, both viral and non-viral, as well as new nucleic acid technologies have led to renewed interest in the field. The field of therapeutic gene editing is rapidly developing with the emergence of CRISPR/Cas9 as well as chemically modified mRNA therapeutics. These new types of nucleic acid therapies are also a good fit with delivery by non-viral delivery approaches which has led to a renewed interest in lipid-based and other nanoformulations.
\end{abstract}




\section{Introduction}

Cystic fibrosis (CF) is a recessively inherited disease caused by mutations in the gene for the cystic fibrosis transmembrane conductance regulator protein (CFTR) located on the long arm of chromosome 7 [1]. CFTR is a membrane protein located on the apical surface of epithelial cells and acts as a channel for anions including chloride and bicarbonate. In CF, the mutated protein results in an imbalance of salt and fluid transport as well as $\mathrm{pH}$ of the airway surface liquids that leads to the production of thick sticky mucus, bacterial infections and inflammatory damage to the lung [1]. Advances in understanding of CFTR mutations and the nature of the protein defect [2] have enabled the development of CFTR modulator drugs such as Ivacaftor, which are effective for patients with rare types of mutations, such as G551D, while other small molecule drugs are now in development for different classes of CFTR mutations [3]. However, the majority of the 281 disease-causing CFTR mutations (https://cftr2.org/mutations_history) remain untreatable with small molecule drugs and so new approaches to gene therapy are under investigation (Figure 1). Advances in nucleic acid technologies, such as CRISPR/Cas9 have opened up new opportunities for both mutation specific and universal gene therapies while new viral and non-viral vectors offer improved delivery. Each approach offers different advantages of persistence and repeatability of administration. (Table 1). This review describes some of the recent developments in genetic therapies of specific relevance for CF.

\section{Viral Gene therapy}

\section{i. Adeno Associated Virus (AAV)}

Previous clinical gene therapy studies for CF using AAV serotype 2 (AAV2) were unsuccessful due to insufficient levels of CFTR transgene expression [4*]. Recent research has focused on 
approaches to expand AAV tropism [6-8], reduce immunogenicity and enhance CFTR expression levels and persistence in the lung (Table 1). These studies exemplify the challenge of developing efficient $\mathrm{AAV}$ vectors for evaluation in relevant CF animal models, including pigs [4*], sheep [7], ferrets [8] and mice [6], but also provide efficient transduction of human epithelia for clinical translation. For example, nine naturally occurring AAV serotypes tested in human cells and murine lungs identified AAV6 as the strongest candidate in both human and mouse epithelium [5]. However, other serotypes, including AAV2/5, i.e. the AAV2 genome packaged in AAV5 capsid proteins [6], were also effective in human cells in vitro and murine lungs in vivo, while AAV8 was identified as the optimal serotype for testing in sheep airways [7].

An alternative approach to expanding AAV tropism, which also overcomes the packaging constrains of AAV, reported using the capsid of human Bocavirus-Type-1 (HBoV1) to package an AAV2 genome [8]. HBoV1, like AAV, is a parvovirus that displays high tropism for the apical membrane of human airway epithelium and transduces human air-liquid interface cultures efficiently. Apart from its tropism, it also has a larger capsid than AAV with a packaging capacity of 5543 nt compared to the 4679 nt of AAV. CF ferrets recapitulate many of the physiological aspects of CF lung disease and are thus proposed to provide a suitable model for the development and testing of CF gene therapy strategies and so rAAV2/HBoV1 vector was evaluated in ferret epithelial models [8]. It was shown that ferret epithelium in vitro and in vivo is permissible to transduction with rAAV2/HBoV1 vectors encoding luciferase reporter genes and that repeated dosing in vivo was effective at maintaining transgene expression [8]. This work opens up the possibility of using CF ferrets to evaluate rAAV2/HBoV1 vectors for CF gene therapy.

Another approach to expanding AAV vector tropism involves directed capsid selection 
methods and this approach was used to identify a novel AAV serotype with specificity and high transduction efficiency for pig airway epithelium to enable testing of therapeutic correction in the gut-corrected CF pig [4*]. This virus packaged a truncated CF gene, CFTR $\Delta R$, and synthetic promoter/enhancer which rescued anion transport and restored bacterial killing after administration to the lungs of CFTR-null pigs. This AAV serotype, however, was specific for pigs so alternative serotypes will be required for man. The use of AAV2/5 encoding the same CFTR $\triangle \mathrm{R}$ minigene with a shortened CMV promoter was reported by other investigators to transduce CF epithelia and restore full channel activities, as measured by human intestinal organoid swelling after forskolin stimulation [6]. Moreover, treatment of a group of CF mice demonstrated, in most individuals, correction of ion transport as shown by the response of their nasal potential differences to low chloride and forskolin perfusion [6].

\section{ii. Lentivirus}

Lentiviral vectors are attractive vectors for CF gene therapy owing to their ability to integrate into the recipient genome, so producing long-term, stable gene expression (Table 1). They have been less well studied than AAV as gene therapy vectors in the lungs, and so less is known about their safety profile or immunogenicity on repeat dosing, while production of high-titre virus preparations is more challenging than for AAV. However, packaging of full length CFTR and strong promoters is not size-limited as with AAV and recent developments, described below, have indicated promising potential for their use in CF gene therapy. As with $\mathrm{AAV}$, one of the major questions concerns viral tropism.

CFTR channel activity was corrected in the airways of CF pigs transduced with a feline immunodeficiency virus (FIV)-based lentiviral vector, pseudotyped with the GP64 envelope protein [9*]. Two weeks after aerosolized FIV-CFTR viral vector delivery to the nose and lung 
of three new-born CF pigs, epithelial tissues revealed a significant increase in transepithelial cAMP-stimulated $\mathrm{Cl}^{-}$current, indicative of functional CFTR. In addition, increases in tracheal airway surface liquid $\mathrm{pH}$ and bacterial killing in CFTR vector-treated animals were observed. Further studies are required to demonstrate the potential of this vector for gene therapy efficacy in treating or preventing lung disease and the ability to redeliver the treatment.

In another format, a simian immunodeficiency virus (SIV)-based lentiviral vector, pseudotyped with Sendai virus fusion protein (F) and Hemagglutinin/ Neuraminidase (HN) envelope proteins was reported to generate efficient transduction of cultured human bronchial epithelial cells and murine lung epithelium in vivo with no detectable immune responses, suggesting that these viral vectors possibly offer unique, non-immunogenic properties [10].

\section{Non-viral Gene Therapy Formulations}

Liposomal vectors for gene therapy offer advantages of safety, ease of scalable formulation and packaging capacity for large DNA molecules. Gene therapy trials for CF were performed with the pGM169/GL67A gene-liposome formulation in a Phase I/Ila clinical study [11], which confirmed safety of the formulation and procedure, and then in a repeat dosing trial in which patients received monthly repeated dosing for up to one year [12]. In terms of efficacy, a number of outcomes showed a trend favouring the treatment group as well as a small but statistically significant increase in lung function measurements at 12 months. The study also demonstrated the safety of repeated dosing, which will be required with most anticipated forms of CF gene therapy, but highlighted the need for improved nucleic acid delivery.

Approaches to enhance transfection efficiency in the lung include equipping them to overcome better the epithelial barriers. For example, DNA nanoparticle formulations of the 
biodegradable poly( $\beta$-amino esters) (PBAEs) polymers covered by a dense layer of polyethylene glycol (PEG) displayed increased penetration of mucus in vitro, which correlated with increased transfection efficiency of murine lungs in vivo. [13]. An interesting, alternative, non-viral approach to CF gene therapy reported the use of exosomes, naturally occurring extracellular vesicles formed by membrane budding from donor cells [14]. Exosomes from CFTR-expressing Calu3 epithelial cells, were incubated with CF15, an epithelial cell line expressing the most common CF mutation F508del-CFTR, and found to transfer both glycosylated exosomal membrane-bound CFTR and CFTR mRNA in the exosomal cytoplasm, restoring CFTR protein production and ion transport functionality to CF15 cells [14].

\section{Improved Nucleic acids for Non-Viral Delivery}

\section{i. Minicircle DNA}

Recent clinical trials of CF gene therapy have employed the plasmid pGM169, which is depleted of inflammatory CpG repeat elements, delivered by the GL67A liposome $[11,12]$. This plasmid previously achieved sustained gene expression in the mouse lung without causing inflammation [15]. However, the Phase I/Ila safety study revealed a dose-dependent, flu-like response to pGM169/GL67A while CFTR transgene mRNA levels in airway epithelial cells were very low [11] or undetectable [12] by quantitative, real-time PCR. The authors attributed the inflammatory response, particularly at higher doses, to the viscous, cationic formulation although the possibility of a DNA-specific response, despite the lack of CpG motifs, was not excluded [11]. This led to the repeat-dosing study being performed with the lowest dose of DNA together with anti-pyretic paracetamol [12]. Thus, for future studies, while improved lipids or other gene carriers would be beneficial, more potent gene expression vectors would enable more robust expression at lower doses, lowering the 
amounts of cationic lipids required for delivery and so potentially reducing inflammatory responses.

Approaches to improving the activity of nucleic acid cargoes delivered by nanoparticles include minicircle DNA, circular pieces of DNA containing the transgene and regulatory elements but from which almost all vestiges of bacterial DNA have been removed, including the antibiotic selection marker. Studies using a lipid-peptide nanoparticle suggested that minicircles offer improved expression levels and duration of expression in human epithelial cells in vitro and in murine epithelia in vivo, along with reduced inflammatory responses [16*]. In another report, however, using polyethylenimine (PEI) as the carrier, murine lung transfections showed that the advantages of the minicircle in transgene expression levels over a plasmid were less evident if the transgene, in this case for luciferase, was additionally CpG-free [17]. Further studies will be required to evaluate whether minicircle DNA delivered by non-viral nanoparticles offers advantages for CF gene therapy over CpGfree plasmid DNA and viral vectors (Table 1).

\section{ii. Oligonucleotides and siRNA}

Approximately $13 \%$ of disease-causing CFTR mutations involve aberrant exon splicing [3] such as the c. $2657+5 G>A$ splicing mutation, which leads to exon 16 skipping or introduction of a premature stop codon $\left[18^{*}\right]$. Single-stranded DNA oligonucleotides of 19 nucleotides containing 2' O-methyl modified ribose and a phosphorothioate backbone were designed that hybridise to pre-mRNA to modify aberrant splicing and were found to restore correct splicing in HEK293 cells expressing the c.2657+5G>A mutant CFTR minigene [18*]. A similar approach could be taken with other such splice mutations as well as those that introduce cryptic splice sites such as the c.3849+10kb C>T mutation (Figure 1). 
Small interfering RNA (siRNA) therapies in development include silencing of the epithelial sodium channel, ENaC. Sodium is the counterion to chloride, and ENaC is upregulated in CF, leading to dehydration of the airway surface liquid (ASL) [19]. Silencing of ENaC mediated by siRNA has proven challenging due to the lack of effective delivery formulations, but an efficient lipid-peptide nanocomplex was described recently that displayed efficient transfection both in vitro and in vivo that may enable siRNA therapy for CF [20].

\section{iii. mRNA Therapy}

Chemically-modified mRNA has been developed as an indirect means of protein replacement with CFTR delivery to the lungs as a therapeutic candidate (Table 1; Figure 1) [21] .Chemically modified mRNAs synthesised in vitro incorporate modified nucleosides that reduce their immunoinflammatory potential and enhance their stability and expression. Compared to DNA encoded gene delivery, mRNA offers higher levels of protein production with greater safety since there is no risk of chromosomal integration. Liposomal or polymeric non-viral vector formulations are essential for mRNA delivery across the epithelial barriers and such formulations often have different characteristics to those used for plasmid DNA delivery [22]. New approaches to developing more efficient nanocarriers for mRNA delivery include lipid library screening $[23,24]$. Because mRNAs have a short half-life in vivo in the lungs of about 7 hours [22], they would require regular, repeated delivery to maintain CFTR expression, which is usually a favourable property of liposomal systems (Table 1). However, mRNA can also be used to deliver Cas9 nuclease for therapeutic gene editing where transient expression is not a limiting factor, but a positive safety factor as described below. 


\section{Gene editing for CF}

Gene editing offers an approach to repair specific mutations in CFTR with great precision, or to disrupt coding sequences in contributory genes, such as ENaC, or cryptic splice sites in CFTR (Figure 1; Table 1). Approaches include Zinc Finger nucleases (ZFNs), Transcription activatorlike effector nucleases (TALENs) and Clustered Regularly Interspersed Palindromic Repeats (CRISPR)/CRISPR-associated nuclease 9 (Cas9), all of which first introduce targeted, double strand breaks in the chromosome near to the mutation site, which are then repaired by the endogenous DNA repair pathways, either by Non-Homologous End-Joining (NHEJ) or, in the presence of a DNA donor, by Homology Directed Repair (HDR) $[25,26]$. ZFN and TALENs are protein targeted and therefore time consuming and expensive to use. By contrast, CRISPR/Cas9 is targeted to a specific chromosomal site by guide RNAs, while the simplicity and low cost of CRISPR has made gene editing a routine tool in most molecular biology labs $[25,26]$.

ZFNs were reported to correct the F508del-CFTR mutation with a selectable plasmid donor in inducible pluripotential stem cells (iPSCs) generated from fibroblasts of a CF donor $\left[27^{*}\right]$. Gene editing restored fully glycosylated CFTR protein production and correction of the ion transport defect. However, mutation specific therapies are problematic for CF as there are at least 281 disease-causing CFTR mutations (https://cftr2.org/mutations_history). An alternative approach to gene editing, termed "super-exon" therapy, has the potential to treat a far wider range of patients as a single therapeutic. ZFNs were used to insert a CFTR cDNA fragment covering exons 11-27, the super-exon, into exon 11, producing functional correction of ion transport characteristics in cell culture [28]. A potential limitation of this approach to 
be determined is whether this may change the relative position of one or more known strong transcriptional enhancers, which may alter the expression of the CFTR gene [29].

Gene editing with targeted nucleases presents risks of off-target DNA cleavage introducing unwanted mutations with unknown consequences. Whole genome sequence may be the best way to detect nuclease-mediated mutations but this is not always practical and so sensitive and unbiased genome-wide methods have been developed to detect offtarget effects including Digenome-seq [30], GUIDEseq [31] and CIRCLE-seq [32]. Efforts to minimise the potential for off-target effects include delivering Cas9 mRNA which has a shorter half-life in vivo than DNA, minimising opportunities for off-target cleavage. This approach was used for in vivo therapy of Surfactant Protein-B (Sp-B) deficiency in a murine model, with CRISPR/Cas9 system delivered by liposomes and the template DNA by AAV6 [33].

Nuclease-free approaches to gene editing would help to overcome these concerns such as the report of triplex-forming Peptide Nucleic Acids (PNAs) that were used to target and repair the F508del-CFTR mutation [34*]. PNAs were delivered with polymeric nanoparticles, achieving correction of CFTR ion transport of up to $25 \%$ in vitro. Another nuclease-free method for CFTR gene editing was described in which helper-dependent Adenoviral (HDAd) vectors, with donor DNAs with regions of homology to the CFTR locus of more than $20 \mathrm{~kb}$, were used to transduce CF iPSCs, achieving levels of correction of more than $50 \%$ with positive selection [35]. The selectable marker was then excised by piggybac transposase leading to "footprintless" gene editing of CFTR. However, the high immunogenicity of adenovirus would limit its utility in vivo. RNA editing approaches are also under development using modified ADAR (Adenosine Deaminase that Acts on RNA) enzymes, in which the catalytic domain is linked to an antisense oligonucleotide [36]. ADAR converts an 
A to a $\mathrm{G}$ in mRNA which might find particular use in correcting premature termination codons and offer fewer off-target effects in the genome compared to Cas9 [36].

\section{Conclusion}

Cystic fibrosis provides one of the finest examples of how a deeper understanding of disease genetics can lead to the development of effective personalised medicines including drugs such as Ivacaftor and Orkambi [37]. Although gene therapy for CF has had a long, and largely disappointing record, deeper understanding of epithelial biology, a renewed effort to develop improved viral and nanoparticle vectors and the development of novel nucleic acid therapeutics, including mRNA and gene editing with CRISPR/Cas9, are opening up new prospects for CF gene therapy. Model systems including CF ferrets and pigs are enabling the development of disease-relevant functional metrics in animal models while improved in vitro human cell models including air-liquid interface cultures and epithelial organoids are now more widely available which are essential for the development of personalised genetic medicines and assessment of their ability to correct the physiological defects of the CF airway epithelium.

\section{Acknowledgements}

Funding: SH and PT were supported by a Cystic Fibrosis Trust Strategic Research Centre grant (2016-SRC006). SH was supported by a Cystic Fibrosis Foundation project grant (HART15XXO) and by the National Institute for Health Research Biomedical Research Centre at Great Ormond Street Hospital for Children NHS Foundation Trust and University College London. 


\section{References}

1. Ratjen F, Bell SC, Rowe SM, Goss CH, Quittner AL, Bush A: Cystic fibrosis. Nat Rev Dis Primers 2015, 1:15010.

2. Cutting GR: Cystic fibrosis genetics: from molecular understanding to clinical application. Nat Rev Genet 2015, 16:45-56.

3. Bell SC, De Boeck K, Amaral MD: New pharmacological approaches for cystic fibrosis: promises, progress, pitfalls. Pharmacol Ther 2015, 145:19-34.

4. Steines B, Dickey DD, Bergen J, Excoffon KJ, Weinstein JR, Li X, Yan Z, Abou Alaiwa $\mathrm{MH}$, Shah VS, Bouzek DC, et al.: CFTR gene transfer with AAV improves early cystic fibrosis pig phenotypes. JCI Insight 2016, 1:e88728.

(*) AAV vector tropism by directed capsid selection was used to identify a novel AAV serotype with specificity and high transduction efficiency for pig airway epithelium. This virus, packaging CFTR $\Delta \mathrm{R}$, rescued anion transport and restored bacterial killing after administration to the lungs of CFTR-null pigs.

5. Kurosaki F, Uchibori R, Mato N, Sehara Y, Saga Y, Urabe M, Mizukami H, Sugiyama $Y$, Kume A: Optimization of adeno-associated virus vector-mediated gene transfer to the respiratory tract. Gene Ther 2017, 24:290-297.

6. Vidovic D, Carlon MS, da Cunha MF, Dekkers JF, Hollenhorst MI, Bijvelds MJ, Ramalho AS, Van den Haute C, Ferrante M, Baekelandt V, et al.: rAAV-CFTR $\Delta \mathbf{R}$ Rescues the Cystic Fibrosis Phenotype in Human Intestinal Organoids and Cystic Fibrosis Mice. Am J Respir Crit Care Med 2016, 193:288-298. 
7. McClain LE, Davey MG, Zoltick PW, Limberis MP, Flake AW, Peranteau WH: Vector serotype screening for use in ovine perinatal lung gene therapy. J Pediatr Surg 2016, 51:879-884.

8. Yan Z, Feng Z, Sun X, Zhang Y, Zou W, Wang Z, Jensen-Cody C, Liang B, Park SY, Qiu J, et al.: Human Bocavirus Type-1 Capsid Facilitates the Transduction of Ferret Airways by Adeno-Associated Virus Genomes. Hum Gene Ther 2017, 28:612-625.

9. Cooney AL, Abou Alaiwa MH, Shah VS, Bouzek DC, Stroik MR, Powers LS, Gansemer ND, Meyerholz DK, Welsh MJ, Stoltz DA, et al.: Lentiviral-mediated phenotypic correction of cystic fibrosis pigs. JCI Insight 2016, 1:e88730.

$\left({ }^{*}\right) \quad$ CFTR channel activity was corrected in the airways of CF pigs transduced with a feline immunodeficiency virus (FIV)-based lentiviral vector, pseudotyped with the GP64 envelope protein. CF pig, epithelial tissues revealed a significant increase in transepithelial cAMP-stimulated $\mathrm{Cl}^{-}$current.

10. Alton EW, Beekman JM, Boyd AC, Brand J, Carlon MS, Connolly MM, Chan M, Conlon S, Davidson HE, Davies JC, et al.: Preparation for a first-in-man lentivirus trial in patients with cystic fibrosis. Thorax 2017, 72:137-147.

11. Alton EW, Boyd AC, Porteous DJ, Davies G, Davies JC, Griesenbach U, Higgins TE, Gill DR, Hyde SC, Innes JA, et al.: A Phase I/Ila Safety and Efficacy Study of Nebulized Liposome-mediated Gene Therapy for Cystic Fibrosis Supports a Multidose Trial. Am J Respir Crit Care Med 2015, 192:1389-1392.

12. Alton EW, Armstrong DK, Ashby D, Bayfield KJ, Bilton D, Bloomfield EV, Boyd AC, Brand J, Buchan R, Calcedo R, et al.: Repeated nebulisation of non-viral CFTR gene therapy in patients with cystic fibrosis: a randomised, double-blind, placebocontrolled, phase $\mathbf{2 b}$ trial. Lancet Respir Med 2015, 3:684-691. 
13. Mastorakos P, da Silva AL, Chisholm J, Song E, Choi WK, Boyle MP, Morales MM, Hanes J, Suk JS: Highly compacted biodegradable DNA nanoparticles capable of overcoming the mucus barrier for inhaled lung gene therapy. Proc Natl Acad Sci U S A 2015, 112:8720-8725.

14. Vituret C, Gallay K, Confort MP, Ftaich N, Matei Cl, Archer F, Ronfort C, Mornex JF, Chanson M, Di Pietro A, et al.: Transfer of the Cystic Fibrosis Transmembrane Conductance Regulator to Human Cystic Fibrosis Cells Mediated by Extracellular Vesicles. Hum Gene Ther 2016, 27:166-183.

15. Hyde SC, Pringle IA, Abdullah S, Lawton AE, Davies LA, Varathalingam A, NunezAlonso G, Green AM, Bazzani RP, Sumner-Jones SG, et al.: CpG-free plasmids confer reduced inflammation and sustained pulmonary gene expression. Nat Biotechnol 2008, 26:549-551.

16. Munye MM, Tagalakis AD, Barnes JL, Brown RE, McAnulty RJ, Howe SJ, Hart SL: Minicircle DNA Provides Enhanced and Prolonged Transgene Expression Following Airway Gene Transfer. Sci Rep 2016, 6:23125.

(*) Minicircles are circular pieces of DNA containing the transgene and regulatory elements but from which almost all vestiges of bacterial DNA have been removed. Transfections showed improved expression levels and duration of expression in human epithelial cells in vitro and in murine epithelia in vivo, along with reduced inflammatory responses.

17. Bazzani RP, Pringle IA, Connolly MM, Davies LA, Sumner-Jones SG, Schleef M, Hyde SC, Gill DR: Transgene sequences free of CG dinucleotides lead to high level, longterm expression in the lung independent of plasmid backbone design. Biomaterials 2016, 93:20-26. 
18. Igreja S, Clarke LA, Botelho HM, Marques L, Amaral MD: Correction of a Cystic Fibrosis Splicing Mutation by Antisense Oligonucleotides. Hum Mutat 2016, 37:209215.

$\left(^{*}\right) \quad$ The c.2657+5G>A mutation CFTR causes aberrant intron splicing with loss of exon 16. Chemically modified, single-stranded DNA oligonucleotides hybridise to premRNA and restore correct splicing of the c. $2657+5 G>A$ mutation in a CFTR minigene.

19. Boucher RC: Cystic fibrosis: a disease of vulnerability to airway surface dehydration. Trends Mol Med 2007, 13:231-240.

20. Manunta MD, Tagalakis AD, Attwood M, Aldossary AM, Barnes JL, Munye MM, Weng A, McAnulty RJ, Hart SL: Delivery of ENaC siRNA to epithelial cells mediated by a targeted nanocomplex: a therapeutic strategy for cystic fibrosis. Sci Rep 2017, 7:700.

21. Alton EW, Boyd AC, Davies JC, Gill DR, Griesenbach U, Harrison PT, Henig N, Higgins T, Hyde SC, Innes JA, et al.: Genetic medicines for CF: Hype versus reality. Pediatr Pulmonol 2016, 51:S5-S17.

22. Pardi N, Tuyishime S, Muramatsu H, Kariko K, Mui BL, Tam YK, Madden TD, Hope MJ, Weissman D: Expression kinetics of nucleoside-modified mRNA delivered in lipid nanoparticles to mice by various routes. J Control Release 2015, 217:345-351.

23. Li B, Luo X, Deng B, Wang J, McComb DW, Shi Y, Gaensler KM, Tan X, Dunn AL, Kerlin BA, et al.: An Orthogonal Array Optimization of Lipid-like Nanoparticles for mRNA Delivery in Vivo. Nano Lett 2015, 15:8099-8107.

24. Oberli MA, Reichmuth AM, Dorkin JR, Mitchell MJ, Fenton OS, Jaklenec A, Anderson DG, Langer R, Blankschtein D: Lipid Nanoparticle Assisted mRNA Delivery for Potent Cancer Immunotherapy. Nano Lett 2017, 17:1326-1335. 
25. Cornu TI, Mussolino C, Cathomen T: Refining strategies to translate genome editing to the clinic. Nat Med 2017, 23:415-423.

26. Harrison PT, Sanz DJ, Hollywood JA: Impact of gene editing on the study of cystic fibrosis. Hum Genet 2016, 135:983-992.

27. Crane AM, Kramer P, Bui JH, Chung WJ, Li XS, Gonzalez-Garay ML, Hawkins F, Liao W, Mora D, Choi S, et al.: Targeted correction and restored function of the CFTR gene in cystic fibrosis induced pluripotent stem cells. Stem Cell Reports 2015, 4:569-577.

(*) Gene editing by Zinc Finger Nucleases was used to correct the F508del-CFTR mutation with a selectable plasmid donor in inducible pluripotential stem cells generated from fibroblasts of a CF donor, restoring fully-glycosylated CFTR protein production and correcting the ion transport defect.

28. Bednarski C, Tomczak K, Vom Hovel B, Weber WM, Cathomen T: Targeted Integration of a Super-Exon into the CFTR Locus Leads to Functional Correction of a Cystic Fibrosis Cell Line Model. PLoS One 2016, 11:e0161072.

29. Ott CJ, Blackledge NP, Kerschner JL, Leir SH, Crawford GE, Cotton CU, Harris A: Intronic enhancers coordinate epithelial-specific looping of the active CFTR locus. Proc Natl Acad Sci U S A 2009, 106:19934-19939.

30. Kim D, Bae S, Park J, Kim E, Kim S, Yu HR, Hwang J, Kim JI, Kim JS: Digenome-seq: genome-wide profiling of CRISPR-Cas9 off-target effects in human cells. Nat Methods 2015, 12:237-243.

31. Zhu L, Lawrence M, Gupta A, Pages H, Kucukural A, Garber M, Wolfe SA: GUIDEseq: a bioconductor package to analyze GUIDE-Seq datasets for CRISPR-Cas nucleases. BMC Genomics 2017, 18:379. 
32. Tsai SQ, Nguyen NT, Malagon-Lopez J, Topkar VV, Aryee MJ, Joung JK: CIRCLE-seq: a highly sensitive in vitro screen for genome-wide CRISPR-Cas9 nuclease off-targets. Nat Methods 2017, 14:607-614.

33. Mahiny AJ, Dewerth A, Mays LE, Alkhaled M, Mothes B, Malaeksefat E, Loretz B, Rottenberger J, Brosch DM, Reautschnig $\mathrm{P}$, et al.: In vivo genome editing using nuclease-encoding mRNA corrects SP-B deficiency. Nat Biotechnol 2015, 33:584586.

34. McNeer NA, Anandalingam K, Fields RJ, Caputo C, Kopic S, Gupta A, Quijano E, Polikoff L, Kong Y: Nanoparticles that deliver triplex-forming peptide nucleic acid molecules correct F508del CFTR in airway epithelium. Nat Commun 2015, 6:6952.

$\left.{ }^{*}\right) \quad$ The paper describes a nuclease-free approach to gene editing using triplex-forming Peptide Nucleic Acids (PNAs) delivered with polymeric nanoparticles to target and repair the F508del-CFTR mutation.

35. Palmer DJ, Grove NC, Ing J, Crane AM, Venken K, Davis BR, Ng P: Homology Requirements for Efficient, Footprintless Gene Editing at the CFTR Locus in Human iPSCs with Helper-dependent Adenoviral Vectors. Mol Ther Nucleic Acids 2016, 5:e372.

36. Montiel-Gonzalez MF, Vallecillo-Viejo IC, Rosenthal JJ: An efficient system for selectively altering genetic information within mRNAs. Nucleic Acids Res 2016, 44:e157.

37. Veit G, Avramescu RG, Chiang AN, Houck SA, Cai Z, Peters KW, Hong JS, Pollard HB, Guggino WB, Balch WE, et al.: From CFTR biology toward combinatorial pharmacotherapy: expanded classification of cystic fibrosis mutations. Mol Biol Cell 2016, 27:424-433. 


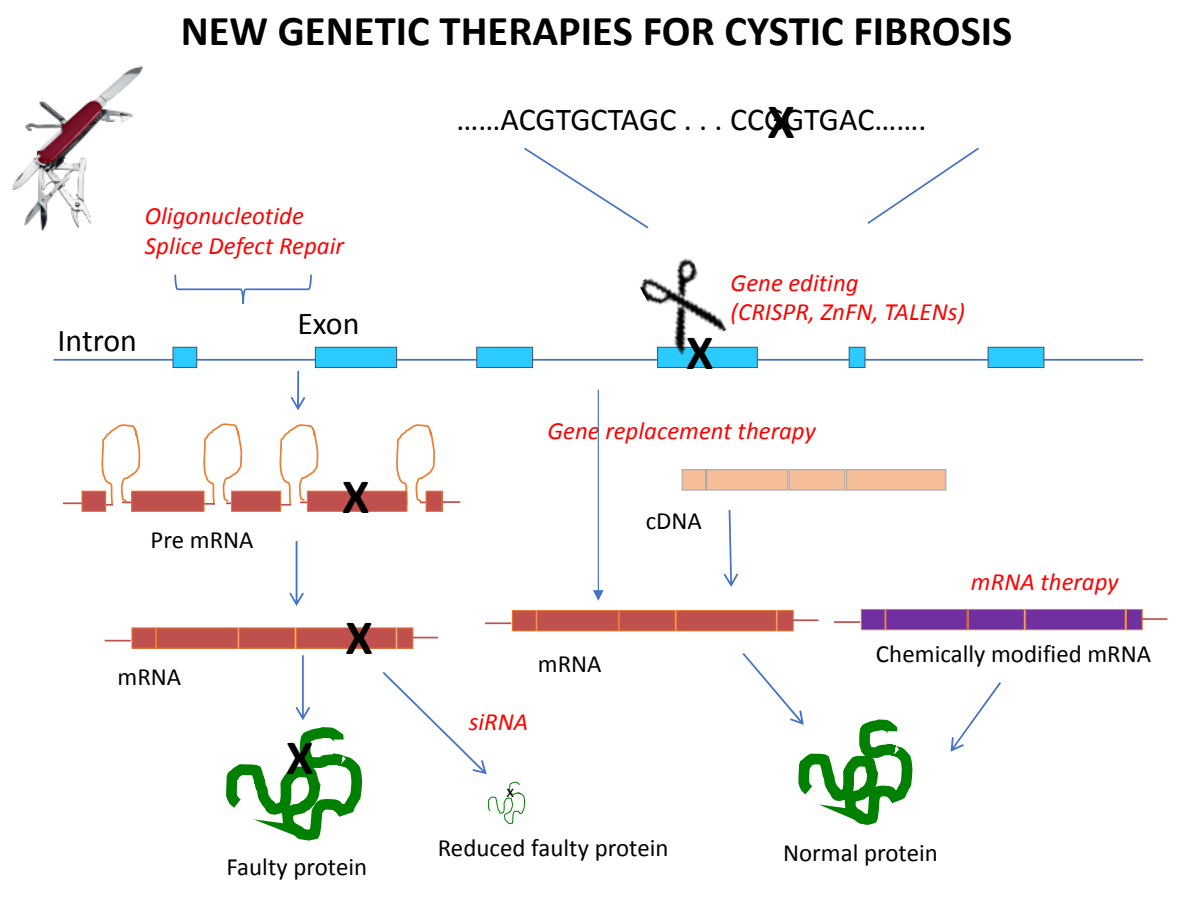

Figure 1

The figure shows the structure of a stylised gene with exons and introns and a disease-causing mutation in one of the exons. The left side of the figure shows that the mutation is transcribed to pre-mRNA and mRNA then translated to a defective protein. The right side of the figure illustrates different molecular approaches to correcting the mutation to either reduce expression, as achieved with siRNA, to replace the defective gene by delivery of a corrected cDNA, by oligonucleotides to correct a splice mutation and finally by gene editing to correct the mutation in the chromosome. 